\title{
Two Temperature Magneto-Thermoelasticity with Initial Stress: State Space Formulation
}

\author{
Sunita Deswal and Kapil Kumar Kalkal \\ Department of Mathematics, G. J. University of Science and Technology, Hisar, Haryana 125001, India \\ Correspondence should be addressed to Kapil Kumar Kalkal; kapilkalkal_gju@rediffmail.com
}

Received 12 May 2013; Accepted 20 August 2013

Academic Editor: Felix Sharipov

Copyright (C) 2013 S. Deswal and K. K. Kalkal. This is an open access article distributed under the Creative Commons Attribution License, which permits unrestricted use, distribution, and reproduction in any medium, provided the original work is properly cited.

\begin{abstract}
Magneto-thermoelastic interactions in an initially stressed isotropic homogeneous elastic half-space with two temperatures are studied using mathematical methods under the purview of the L-S model of linear theory of generalized thermoelasticity. The formalism deals with the state space approach with the purpose of counteracting the difficulties of handling the displacement potential functions. Of specific concern here is the propagation of waves owing to ramp type increase in temperature and load. The medium is considered to be permeated by a uniform magnetic field. The expressions for different field parameters such as displacement, temperature, strain, and stress in the physical domain are obtained by applying a numerical inversion technique. Results of some earlier workers have been deduced from the present formulation. Numerical work is also performed for a suitable material with the aim of illustrating the results.
\end{abstract}

\section{Introduction}

The classical coupled thermoelasticity theory proposed by Biot [1] with the introduction of the strain-rate term in the Fourier heat conduction equation leads to a parabolictype heat conduction equation, called the diffusion equation. This theory predicts finite propagation speed for elastic wave but an infinite speed for thermal disturbance. This is physically unrealistic. To overcome such an absurdity, generalized thermoelasticity theories have been propounded by Lord and Shulman [2] as well as Green and Lindsay [3] advocating the existence of finite thermal wave speed in solids. These theories have been developed by introducing one or two relaxation times in the thermoelastic process, either by modifying Fourier's heat conduction equation or by correcting the energy equation and Neumann-Duhamel relation. According to these generalized theories, heat propagation can be visualized as a wave phenomenon rather than a diffusion one; in the literature, it is usually referred to as the second sound effect. These two theories are structurally different from one another, and one cannot be obtained as a particular case of the other. Various problems characterizing these theories have been investigated and have revealed some interesting phenomenon. Brief reviews of this topic have been reported by Chandrasekharaiah $[4,5]$.

The interplay of the Maxwell electromagnetic field with the motion of deformable solids is largely being undertaken by many investigators owing to the possibility of its application to geophysical problems and certain topics in optics and acoustics. Moreover, the earth is subject to its own magnetic field, and the material of the earth may be electrically conducting. Thus, the magnetoelastic nature of the earth's material may affect the propagation of waves. Many authors have considered the propagation of electromagneto-thermoelastic waves in an electrically and thermally conducting solid. A comprehensive review of the earlier contributions to the subject can be found in the study by Paria [6]. Among the authors who considered the generalized magneto-thermoelastic equations are Nayfeh and Nemat-Nasser [7] who studied the propagation of plane waves in a solid under the influence of an electromagnetic field. They have obtained the governing equations in the general case and the solution for some particular cases. Choudhuri [8] extended these results to rotating media. Ezzat [9] has studied the problem of generation of generalized magneto-thermoelastic waves by thermal shock in a perfectly 
conducting half-space. Ezzat et al. [10] have established the model of two-dimensional equations of generalized magneto-thermoelasticity.

In dealing with classical or generalized thermoelastic problems in most situations, the displacement potential function approach is used. However, Bahar and Hetnarski [11] outlined several disadvantages of the potential function approach. These may be summarized in the fact that the boundary and initial conditions of the problem are not related directly to the potential function, as it has no physical meaning explicitly. Secondly, more stringent assumptions must be made on the behaviour of potential functions than on the actual physical quantities. Finally, it was found that many integral representations of physical quantities are convergent in the classical sense, while their potential function representations only converge in the mean. To get rid of these difficulties, Bahar and Hetnarski [11] introduced the state space formulation in thermoelastic problems. This state space approach has been further developed in Sherief [12] to include the effect of heat sources. He et al. [13] surveyed a one-dimensional thermal shock problem for a semi-infinite piezoelectric rod using state space approach. In a survey article, Ezzat [14] has discussed the developments in the theory of thermoelasticity and fluid mechanics. He has also reviewed the method of matrix exponential, which constitutes the basis of the state space approach in the same work. Youssef and El-Bary [15] put forward an analysis for a generalized thermoelastic infinite layer problem under three theories using state space approach. State space formulation to the vibration of gold nanobeam in femtoseconds scale was done by Elsibai and Youssef [16]. Recently, Deswal et al. [17] investigated the effects of magnetic field and initial stress on a fractional order generalized thermoelastic half-space.

The development of initial stresses in the medium is due to many reasons, for example, resulting from variation of temperature, process of quenching, overburden layer, and slow process of creep, gravitation, weight, largeness, and so forth. The earth is supposed to be under high initial stresses. It is therefore of great interest to study the influence of these stresses on the propagation of stress waves. It was the achievement of Biot [18] to show that acoustic propagation under initial stresses would be fundamentally different from that under stress free state and could not be represented merely by introducing stress dependent elastic coefficients in the classical theory. He has obtained the velocities of longitudinal and transverse waves propagating along the coordinate axes only. Many authors have contributed their efforts to study the wave propagation in solids under initial stresses for various models. The study of reflection and refraction phenomenon of plane waves in an unbounded medium under initial stresses is due to Chattopadhyay et al. [19]. Montanaro [20] constructed the model of isotropic linear thermoelasticity with hydrostatic initial stress. Othman and Song [21] and Singh [22] studied the reflection of thermoelastic waves from a free surface under hydrostatic initial stress in the context of different theories of generalized thermoelasticity.

The theory of heat conduction in a deformable body, formulated by Chen and Gurtin [23] and Chen et al. [24, 25] depends on two different temperatures: the conductive temperature $\phi$ and the thermodynamical temperature $T$. Chen et al. [24] have suggested that the difference between these two temperatures is proportional to heat supply. In absence of heat supply, these two temperatures are identical for time independent situation. However, for time dependent cases, particularly for problems related to wave propagation, two temperatures are in general different, regardless of heat supply. The two-temperature thermoelasticity theory has gained much attention of the researchers in the recent years. The existence, structural stability, convergence, and spatial behaviour of two-temperature thermoelasticity have been provided by Quintanilla [26]. Youssef [27] has developed a new model of generalized thermoelasticity that depends on two temperatures $T$ and $\phi$, where the difference between the two temperatures is proportional to heat supply $\phi_{, i i}$ with a nonnegative constant $a$ (length $^{2}$ ). Later on, Youssef and Al-Lehaibi [28] and Youssef and El-Bary [29] investigated various problems on the basis of two temperature thermoelasticity with relaxation times and showed that the obtained results are qualitatively different as compared to those in case of one-temperature thermoelasticity.

It is worthwhile mentioning here that, in most of the earlier studies, mechanical or thermal loading on the bounding surface is considered to be in the form of a shock. But the sudden change in the load is definitely an idealized situation as it is difficult to interpret physically a pulse represented mathematically by a step function; even a very rapid risetime (of the order of $10^{-9}$ second) may be slow in continuum terms. This is particularly true in the case of second sound effects when the thermal relaxation times for typical metals are less than $10^{-9}$ second. It is thus felt that a finite time of rise of external load applied on the bounding surface should be considered while studying a practical problem of this nature. Considering this vital aspect of rise of time, Misra et al. [30] and Youssef with many authors investigated many applications in which the ramp type heating is used $[15,16]$.

The present investigation is intended to demonstrate the use of state space approach in analyzing the propagation of magneto-thermoelastic waves with hydrostatic initial stress in two-temperature theory of thermoelasticity. Laplace transform technique is employed to obtain the general solution. The inverse Laplace transform is carried out numerically using a method based on Fourier expansion technique in Honig and Hirdes [31]. Numerical values of the field quantities are calculated by considering illustrative examples, and their variations with respect to space coordinate are displayed graphically. Some particular cases have also been deduced from the present analysis.

\section{Formulation of the Problem}

Let us consider a perfectly conducting thermoelastic halfspace $x \geq 0$ with hydrostatic initial stress and two temperatures of a linear, homogeneous, and isotropic medium whose state can be expressed in terms of the space variable $x$ and the time variable $t$. The initial conditions are assumed to be homogeneous. The adjacent free space is assumed to be permeated by a uniform magnetic field $\mathbf{H}\left(0, H_{0}, 0\right)$ acting 
parallel to the boundary $x=0$. This produces an induced magnetic field $\mathbf{h}\left(0, h_{2}, 0\right)$ and induced electric field $\mathbf{E}\left(0,0, E_{3}\right)$ which satisfy the linearized equations of electromagnetism and are valid for slowly moving media:

$$
\begin{gathered}
\operatorname{curl} \mathbf{h}=\mathbf{J}+\varepsilon_{0} \frac{\partial \mathbf{E}}{\partial t}, \\
\operatorname{curl} \mathbf{E}=-\mu_{0} \frac{\partial \mathbf{h}}{\partial t}, \\
\mathbf{E}=-\mu_{0}\left(\frac{\partial \mathbf{h}}{\partial t} \times \mathbf{H}\right), \\
\operatorname{div} \mathbf{h}=0 .
\end{gathered}
$$

The above equations are supplemented by the field equations and constitutive relations with initial stress and two temperatures according to Lord and Shulman [2], Montanaro [20], and Youssef [27] as follows.

(1) Constitutive relations:

$$
\begin{gathered}
\sigma_{i j}=-p\left(\delta_{i j}+\omega_{i j}\right)+2 \bar{\mu} e_{i j}+\bar{\lambda} e \delta_{i j}-\beta_{1}\left(T-T_{0}\right) \delta_{i j}, \\
e_{i j}=\frac{1}{2}\left(u_{i, j}+u_{j, i}\right), \\
\omega_{i j}=\frac{1}{2}\left(u_{j, i}-u_{i, j}\right) .
\end{gathered}
$$

(2) Heat conduction equation:

$$
k \phi_{i i}=\rho c_{E}\left(\dot{T}+\tau_{0} \ddot{T}\right)+\beta_{1} T_{0}\left(\dot{e}+\tau_{0} \ddot{e}\right) .
$$

(3) Equation of motion:

$$
\sigma_{j i, j}+F_{i}=\rho \ddot{u}_{i},
$$

where

$$
F_{i}=\mu_{0} \mathbf{J} \times \mathbf{H}
$$

(4) Relation between conductive temperature and thermodynamical temperature:

$$
\phi-T=a \phi, i i .
$$

The displacement components for one-dimensional medium have the form

$$
u_{x}=u(x, t), \quad u_{y}=u_{z}=0 .
$$

The strain component takes the form

$$
e=e_{x x}=\frac{\partial u}{\partial x}
$$

The constitutive equation, heat conduction equation, and equation of motion assume the shape

$$
\begin{gathered}
\sigma=\sigma_{x x}=(\bar{\lambda}+2 \bar{\mu}) e-\beta_{1}\left(T-T_{0}\right)-p, \\
k \frac{\partial^{2} \phi}{\partial x^{2}}=\rho c_{E}\left(\dot{T}+\tau_{0} \ddot{T}\right)+\beta_{1} T_{0}\left(\dot{e}+\tau_{0} \ddot{e}\right), \\
\rho \ddot{u}=\frac{\partial \sigma}{\partial x}+F_{x},
\end{gathered}
$$

with

$$
F_{x}=-\varepsilon_{0} \mu_{0}^{2} H_{0}^{2} \ddot{u}
$$

Equation (10) can be rewritten as

$$
\frac{\partial^{2} \sigma}{\partial x^{2}}=\rho\left(1+\frac{\varepsilon_{0} \mu_{0}^{2} H_{0}^{2}}{\rho}\right) \ddot{e} .
$$

The relation between the conductive temperature and thermodynamical temperature takes the form

$$
\phi-T=a \frac{\partial^{2} \phi}{\partial x^{2}} .
$$

In order to nondimensionalize (9), (12), and (13), let us define the following set of dimensionless variables:

$$
\begin{gathered}
\left(x^{\prime}, u^{\prime}\right)=c_{0} \eta_{0}(x, u), \\
\left(t^{\prime}, \tau_{0}^{\prime}\right)=c_{0}^{2} \eta_{0}\left(t, \tau_{0}\right), \\
\left(\theta, \phi^{\prime}\right)=\frac{1}{T_{0}}\left(T-T_{0}, \phi-T_{0}\right), \\
\sigma^{\prime}=\frac{1}{(\bar{\lambda}+2 \bar{\mu})} \sigma, \quad p^{\prime}=\frac{p}{\beta_{1} T_{0}},
\end{gathered}
$$

where $\eta_{0}=\rho c_{E} / k$ and $c_{0}^{2}=(\bar{\lambda}+2 \bar{\mu}) / \rho$.

Introducing (14) in (9), (12), and (13), we obtain the following equations in nondimensional forms (dropping the primes):

$$
\sigma=e-\varepsilon_{1} \theta-\delta_{1},
$$$$
\frac{\partial^{2} \phi}{\partial x^{2}}=\left(\frac{\partial}{\partial t}+\tau_{0} \frac{\partial^{2}}{\partial t^{2}}\right)(\theta+\varepsilon e),
$$

$$
\begin{gathered}
\frac{\partial^{2} \sigma}{\partial x^{2}}=\alpha \ddot{e}, \\
\phi-\theta=\beta \frac{\partial^{2} \phi}{\partial x^{2}},
\end{gathered}
$$

where $\alpha=1+\varepsilon_{0} \mu_{0}^{2} H_{0}^{2} / \rho, \beta=a c_{0}^{2} \eta_{0}^{2}, \varepsilon=\beta_{1} / \rho c_{E}, \varepsilon_{1}=$ $\beta_{1} T_{0} /(\bar{\lambda}+2 \bar{\mu})$, and $\delta_{1}=p \varepsilon_{1}$ are the coupling parameters. to

Equations (15) will change in the Laplace transform space

$$
\begin{gathered}
\bar{\sigma}=\bar{e}-\varepsilon_{1} \bar{\theta}-\frac{\delta_{1}}{s}, \\
D^{2} \bar{\phi}=\left(s+\tau_{0} s^{2}\right) \bar{\theta}+\left(s+\tau_{0} s^{2}\right) \varepsilon \bar{e} \\
D^{2} \bar{\sigma}=\alpha s^{2} \bar{e} \\
\bar{\phi}-\bar{\theta}=\beta D^{2} \bar{\phi}
\end{gathered}
$$

where $D \equiv \partial / \partial x$, and $s$ denotes the Laplace transform variable (bar over a symbol indicates the quantity in Laplace transform domain). 
Elimination of $\bar{e}$ and $\bar{\theta}$ from (16) and (17) yields

$$
\begin{aligned}
& D^{2} \bar{\phi}=L_{1} \bar{\phi}+L_{2} \bar{\sigma}+L_{3}, \\
& D^{2} \bar{\sigma}=M_{1} \bar{\phi}+M_{2} \bar{\sigma}+M_{3},
\end{aligned}
$$

where

$$
\begin{gathered}
L_{1}=\frac{\left(s+\tau_{0} s^{2}\right)\left(1+\varepsilon \varepsilon_{1}\right)}{\left[1+\beta\left(s+\tau_{0} s^{2}\right)\left(1+\varepsilon \varepsilon_{1}\right)\right]}, \\
L_{2}=\frac{\varepsilon\left(s+\tau_{0} s^{2}\right)}{\left[1+\beta\left(s+\tau_{0} s^{2}\right)\left(1+\varepsilon \varepsilon_{1}\right)\right]}, \\
L_{3}=\frac{\delta_{1} \varepsilon\left(1+\tau_{0} s\right)}{\left[1+\beta\left(s+\tau_{0} s^{2}\right)\left(1+\varepsilon \varepsilon_{1}\right)\right]}, \\
M_{2}=\alpha s^{2}\left(1-\varepsilon_{1} \beta L_{2}\right), \quad M_{3}=\alpha s\left(\delta_{1}-s \beta \varepsilon_{1} L_{3}\right) .
\end{gathered}
$$

Having chosen conductive temperature $\bar{\phi}$ and stress component $\bar{\sigma}$ as the state variables, (18) may be recasted in the form

$$
\frac{d^{2}}{d x^{2}} \bar{V}(x, s)=A(s) \bar{V}(x, s)+B(x, s)
$$

where

$$
\begin{gathered}
A(s)=\left[\begin{array}{cc}
L_{1} & L_{2} \\
M_{1} & M_{2}
\end{array}\right], \quad \bar{V}(x, s)=\left[\begin{array}{l}
\bar{\phi}(x, s) \\
\bar{\sigma}(x, s)
\end{array}\right], \\
B(x, s)=\left[\begin{array}{c}
L_{3} \\
M_{3}
\end{array}\right] .
\end{gathered}
$$

The formal solution of the differential equation (20) can be expressed as

$$
\begin{aligned}
\bar{V}(x, s)= & \exp [-\sqrt{A(s)} x]\left[\bar{V}(0, s)+A^{-1}(s) B(0, s)\right] \\
& -A^{-1}(s) B(x, s),
\end{aligned}
$$

with

$$
\bar{V}(0, s)=\left[\begin{array}{l}
\bar{\phi}(0, s) \\
\bar{\sigma}(0, s)
\end{array}\right]=\left[\begin{array}{l}
\bar{\phi}_{0} \\
\bar{\sigma}_{0}
\end{array}\right]
$$

after cancelling the exponential having positive power to get bounded solution for large $x$.

Now we have to find out the form of the matrix $\exp [-\sqrt{A(s)} x]$. The characteristic equation of the matrix $A(s)$ is obtained as

$$
\lambda^{2}-\left(L_{1}+M_{2}\right) \lambda+\left(L_{1} M_{2}-M_{1} L_{2}\right)=0,
$$

where $\lambda_{1}$ and $\lambda_{2}$ are the roots of (24) which satisfy the relations

$$
\begin{aligned}
\lambda_{1}+\lambda_{2} & =L_{1}+M_{2}, \\
\lambda_{1} \lambda_{2} & =L_{1} M_{2}-M_{1} L_{2} .
\end{aligned}
$$

Now, we can write the spectral decomposition of $A(s)$ as follows, Charles [32]

$$
A(s)=\lambda_{1} F_{1}+\lambda_{2} F_{2},
$$

where $\left(F_{1}, F_{2}\right)$ is a matrix resolution of the identity; that is,

$$
F_{1}^{2}=F_{1}, \quad F_{2}^{2}=F_{2}, \quad F_{1} F_{2}=F_{2} F_{1}=O, \quad F_{1}+F_{2}=I .
$$

Then we have

$$
[A(s)]^{n}=\lambda_{1}^{n} F_{1}+\lambda_{2}^{n} F_{2},
$$

where

$$
\begin{aligned}
& F_{1}=\frac{A-\lambda_{2} I}{\lambda_{1}-\lambda_{2}}=\frac{1}{\left(\lambda_{1}-\lambda_{2}\right)}\left[\begin{array}{cc}
L_{1}-\lambda_{2} & L_{2} \\
M_{1} & M_{2}-\lambda_{2}
\end{array}\right], \\
& F_{2}=\frac{\lambda_{1} I-A}{\lambda_{1}-\lambda_{2}}=\frac{1}{\left(\lambda_{1}-\lambda_{2}\right)}\left[\begin{array}{cc}
\lambda_{1}-L_{1} & -L_{2} \\
-M_{1} & \lambda_{1}-M_{2}
\end{array}\right] .
\end{aligned}
$$

In particular, if $n=1 / 2$, we have

$$
\sqrt{A(s)}=\sqrt{\lambda_{1}} F_{1}+\sqrt{\lambda_{2}} F_{2} .
$$

Finally, we get

$B(s)=\sqrt{A(s)}=\frac{1}{\sqrt{\lambda_{1}}+\sqrt{\lambda_{2}}}\left[\begin{array}{cc}\sqrt{\lambda_{1} \lambda_{2}}+L_{1} & L_{2} \\ M_{1} & M_{2}+\sqrt{\lambda_{1} \lambda_{2}}\end{array}\right]$.

Now the Taylor series expansion of $\exp [-\sqrt{A(s)} x]$ has the form

$$
\Gamma(x, s)=\exp [-\sqrt{A(s)} x]=\exp [-B(s) x]=\sum_{n=0}^{\infty} \frac{[-B(s) x]^{n}}{n !} .
$$

Using the well-known Cayley-Hamilton theorem, we can express $B^{2}$ and higher orders of the matrix $B$ in terms of $I$ and $B$, where $I$ is the unit matrix of order 2 . Thus, the infinite series in (32) can be truncated as

$$
\Gamma(x, s)=\exp [-B(s) x]=b_{0} I+b_{1} B(s),
$$

where $b_{0}$ and $b_{1}$ are some parameters depending on $s$ and $x$.

The characteristic equation of the matrix $B(s)$ can be written as

$$
P^{2}-P\left(\sqrt{\lambda_{1}}+\sqrt{\lambda_{2}}\right)+\sqrt{\lambda_{1} \lambda_{2}}=0 .
$$

The roots of this equation, namely, $P_{1}$ and $P_{2}$, take the forms

$$
P_{1}=\sqrt{\lambda_{1}}, \quad P_{2}=\sqrt{\lambda_{2}} .
$$

According to Cayley-Hamilton theorem, the characteristic roots $P_{1}$ and $P_{2}$ of the matrix $B$ must satisfy (33). Therefore, we get

$$
\begin{aligned}
& \exp \left(-P_{1} x\right)=b_{0}+b_{1} P_{1}, \\
& \exp \left(-P_{2} x\right)=b_{0}+b_{1} P_{2} .
\end{aligned}
$$


The solution of this system of linear equations is given by

$$
\begin{gathered}
b_{0}=\frac{P_{1} e^{-P_{2} x}-P_{2} e^{-P_{1} x}}{P_{1}-P_{2}}, \\
b_{1}=\frac{e^{-P_{1} x}-e^{-P_{2} x}}{P_{1}-P_{2}} .
\end{gathered}
$$

Plugging the above expressions along with $I$ and $B$ into (33), we obtain the components $\Gamma_{i j}(i, j=1,2)$ of the matrix $\Gamma(x, s)$ as

$$
\begin{gathered}
\Gamma_{11}=\frac{e^{-\sqrt{\lambda_{2}} x}\left(\lambda_{1}-L_{1}\right)-e^{-\sqrt{\lambda_{1}} x}\left(\lambda_{2}-L_{1}\right)}{\lambda_{1}-\lambda_{2}}, \\
\Gamma_{12}=\frac{L_{2}\left(e^{-\sqrt{\lambda_{1}} x}-e^{-\sqrt{\lambda_{2}} x}\right)}{\lambda_{1}-\lambda_{2}}, \\
\Gamma_{21}=\frac{M_{1}\left(e^{-\sqrt{\lambda_{1}} x}-e^{-\sqrt{\lambda_{2}} x}\right)}{\lambda_{1}-\lambda_{2}}, \\
\Gamma_{22}=\frac{e^{-\sqrt{\lambda_{2}} x}\left(\lambda_{1}-M_{2}\right)-e^{-\sqrt{\lambda_{1}} x}\left(\lambda_{2}-M_{2}\right)}{\lambda_{1}-\lambda_{2}} .
\end{gathered}
$$

Hence solution in (22) can be written as

$$
\bar{V}(x, s)=\Gamma_{i j}\left[\bar{V}(0, s)+A^{-1}(s) B(0, s)\right]-A^{-1}(s) B(x, s) .
$$

Inserting the values of $\bar{V}(x, s), A^{-1}(s)$, and $B(x, s)$ into (39) and after straight-forward calculation, the expressions for conductive temperature and stress are evaluated as

$$
\begin{aligned}
& \bar{\phi}=\frac{1}{\left(\lambda_{1}-\lambda_{2}\right)}\left[\left(\lambda_{1} \bar{\phi}_{0}-L_{1} \bar{\phi}_{0}+\alpha_{1} \lambda_{1}\right.\right. \\
& \left.-\alpha_{1} L_{1}-L_{2} \bar{\sigma}_{0}-\alpha_{2} L_{2}\right) e^{-\sqrt{\lambda_{2}} x} \\
& -\left(\lambda_{2} \bar{\phi}_{0}-L_{1} \bar{\phi}_{0}+\alpha_{1} \lambda_{2}-\alpha_{1} L_{1}\right. \\
& \left.\left.-L_{2} \bar{\sigma}_{0}-\alpha_{2} L_{2}\right) e^{-\sqrt{\lambda_{1}} x}\right]-\alpha_{1}, \\
& \bar{\sigma}=\frac{1}{\left(\lambda_{1}-\lambda_{2}\right)}\left[\left(\lambda_{1} \bar{\sigma}_{0}-M_{1} \bar{\phi}_{0}-\alpha_{1} M_{1}-M_{2} \bar{\sigma}_{0}\right.\right. \\
& \left.+\alpha_{2} \lambda_{1}-\alpha_{2} M_{2}\right) e^{-\sqrt{\lambda_{2}} x} \\
& -\left(\lambda_{2} \bar{\sigma}_{0}-M_{2} \bar{\sigma}_{0}-M_{1} \bar{\phi}_{0}-\alpha_{1} M_{1}\right. \\
& \left.\left.+\alpha_{2} \lambda_{2}-\alpha_{2} M_{2}\right) e^{-\sqrt{\lambda_{1}} x}\right]-\alpha_{2},
\end{aligned}
$$

where

$$
\begin{gathered}
\alpha_{1}=\frac{T_{1}}{T_{3}}, \quad \alpha_{2}=\frac{T_{2}}{T_{3}}, \\
T_{1}=M_{2} L_{3}-L_{2} M_{3}, \quad T_{2}=L_{1} M_{3}-M_{1} L_{3}, \\
T_{3}=L_{1} M_{2}-M_{1} L_{2} .
\end{gathered}
$$

Combination of (17) and (40) yields

$$
\begin{aligned}
\bar{\theta}=\frac{1}{\left(\lambda_{1}-\lambda_{2}\right)}[ & \left(\lambda_{1} \bar{\phi}_{0}-L_{1} \bar{\phi}_{0}+\alpha_{1} \lambda_{1}-\alpha_{1} L_{1}\right. \\
& \left.-L_{2} \bar{\sigma}_{0}-\alpha_{2} L_{2}\right)\left(1-\beta \lambda_{2}\right) e^{-\sqrt{\lambda_{2}} x} \\
& -\left(\lambda_{2} \bar{\phi}_{0}-L_{1} \bar{\phi}_{0}+\alpha_{1} \lambda_{2}-\alpha_{1} L_{1}\right. \\
& \left.\left.-L_{2} \bar{\sigma}_{0}-\alpha_{2} L_{2}\right)\left(1-\beta \lambda_{1}\right) e^{-\sqrt{\lambda_{1}} x}\right]-\alpha_{1}
\end{aligned}
$$

\section{Application}

3.1. Problem I: Ramp Type Increase in Boundary Temperature of an Elastic Half-Space. We consider a homogeneous isotropic thermoelastic solid occupying the half-space $x \geq 0$. The boundary plane $x=0$ of the half space is subject to a ramp type increase in temperature. Mathematically, these boundary conditions can be written as

$$
\begin{gathered}
\sigma(0, t)=-p, \\
\phi(0, t)=\phi^{*} h(t),
\end{gathered}
$$

where $\phi^{*}$ is a constant temperature and $h(t)$ is a function of $t$ defined as

$$
h(t)= \begin{cases}0, & t \leq 0 \\ \frac{t}{t_{0},} & 0<t \leq t_{0} \\ 1, & t \geq t_{0}\end{cases}
$$

where $t_{0}$ is a fixed moment of time.

Employing (14) and Laplace transform on (44) and (45), one can find

$$
\begin{gathered}
\bar{\sigma}(0, s)=\bar{\sigma}_{0}=-\frac{\delta_{1}}{s}, \\
\bar{\phi}(0, s)=\bar{\phi}_{0}=\frac{\phi^{*}\left(1-e^{-s t_{0}}\right)}{t_{0} s^{2}} .
\end{gathered}
$$

We can now use the values of $\bar{\sigma}_{0}$ and $\bar{\phi}_{0}$ from (46) to finally get the solutions in the Laplace transform domain as

$$
\begin{aligned}
\bar{\phi}=\frac{1}{\left(\lambda_{1}-\lambda_{2}\right)}[\{ & \left(\lambda_{1}-L_{1}\right)\left(\alpha_{1}+\phi^{*} \alpha_{3}\right) \\
& \left.-L_{2}\left(\alpha_{2}-\frac{\delta_{1}}{s}\right)\right\} e^{-\sqrt{\lambda_{2}} x} \\
- & \left\{\left(\lambda_{2}-L_{1}\right)\left(\alpha_{1}+\phi^{*} \alpha_{3}\right)\right. \\
- & \left.\left.L_{2}\left(\alpha_{2}-\frac{\delta_{1}}{s}\right)\right\} e^{-\sqrt{\lambda_{1}} x}\right]-\alpha_{1},
\end{aligned}
$$




$$
\begin{aligned}
\bar{\sigma}=\frac{1}{\left(\lambda_{1}-\lambda_{2}\right)}[ & \left\{\left(\lambda_{1}-M_{2}\right)\left(\alpha_{2}-\frac{\delta_{1}}{s}\right)\right. \\
& \left.-M_{1}\left(\alpha_{1}+\alpha_{3} \phi^{*}\right)\right\} e^{-\sqrt{\lambda_{2}} x} \\
& -\left\{\left(\lambda_{2}-M_{2}\right)\left(\alpha_{2}-\frac{\delta_{1}}{s}\right)\right. \\
\bar{\theta}=\frac{1}{\left(\lambda_{1}-\lambda_{2}\right)}\left[\left\{\left(\lambda_{1}-L_{1}\right)\left(\alpha_{1}+\phi^{*} \alpha_{3}\right)\right.\right. & \left.\left.-M_{1}\left(\alpha_{1}+\alpha_{3} \phi^{*}\right)\right\} e^{-\sqrt{\lambda_{1}} x}\right]-\alpha_{2}, \\
& \left.-L_{2}\left(\alpha_{2}-\frac{\delta_{1}}{s}\right)\right\}\left(1-\beta \lambda_{2}\right) e^{-\sqrt{\lambda_{2}} x} \\
& -\left\{\left(\lambda_{2}-L_{1}\right)\left(\alpha_{1}+\phi^{*} \alpha_{3}\right)\right. \\
& \left.\left.-L_{2}\left(\alpha_{2}-\frac{\delta_{1}}{s}\right)\right\}\left(1-\beta \lambda_{1}\right) e^{-\sqrt{\lambda_{1}} x}\right]-\alpha_{1},
\end{aligned}
$$

$$
\begin{aligned}
\bar{e}=\frac{1}{\left(\lambda_{1}-\lambda_{2}\right)}[\{ & \left(\alpha_{1}+\alpha_{3} \phi^{*}\right) \\
& \times\left\{\varepsilon_{1}\left(\lambda_{1}-L_{1}\right)\left(1-\beta \lambda_{2}\right)-M_{1}\right\} \\
+ & \left(\lambda_{1}-M_{2}\right)\left(\alpha_{2}-\frac{\delta_{1}}{s}\right) \\
- & \left.\varepsilon_{1} L_{2}\left(\alpha_{2}-\frac{\delta_{1}}{s}\right)\left(1-\beta \lambda_{2}\right)\right\} e^{-\sqrt{\lambda_{2}} x} \\
- & \left(\alpha_{1}+\alpha_{3} \phi^{*}\right) \\
& \times\left\{\varepsilon_{1}\left(\lambda_{2}-L_{1}\right)\left(1-\beta \lambda_{1}\right)-M_{1}\right\} \\
& +\left(\lambda_{2}-M_{2}\right)\left(\alpha_{2}-\frac{\delta_{1}}{s}\right) \\
& \left.\left.-\varepsilon_{1} L_{2}\left(\alpha_{2}-\frac{\delta_{1}}{s}\right)\left(1-\beta \lambda_{1}\right)\right\} e^{-\sqrt{\lambda_{1}} x}\right] \\
- & \varepsilon_{1} \alpha_{1}-\alpha_{2}+\frac{\delta_{1}}{s},
\end{aligned}
$$

where

$$
\alpha_{3}=\frac{1-e^{-s t_{0}}}{t_{0} s^{2}} .
$$

Considering (10) along with (11) and using nondimensional variables and Laplace transform, the displacement component is evaluated as

$$
\bar{u}=\frac{1}{\alpha s^{2}} \frac{\partial \bar{\sigma}}{\partial x} .
$$

Substituting from (48) into (52), we find

$$
\begin{aligned}
\bar{u}=-\frac{1}{\alpha s^{2}\left(\lambda_{1}-\lambda_{2}\right)}\left[\sqrt { \lambda _ { 2 } } \left\{\left(\lambda_{1}-M_{2}\right)\left(\alpha_{2}-\frac{\delta_{1}}{s}\right)\right.\right. \\
\left.-M_{1}\left(\alpha_{1}+\alpha_{3} \phi^{*}\right)\right\} e^{-\sqrt{\lambda_{2}} x} \\
-\sqrt{\lambda_{1}}\left\{\left(\lambda_{2}-M_{2}\right)\left(\alpha_{2}-\frac{\delta_{1}}{s}\right)\right. \\
\left.\left.-M_{1}\left(\alpha_{1}+\alpha_{3} \phi^{*}\right)\right\} e^{-\sqrt{\lambda_{1}} x}\right] .
\end{aligned}
$$

3.2. Problem II: Ramp Type Increase in Boundary Load of an Elastic Half-Space. In this case we assume that the plane boundary of the half-space $x \geq 0$ is kept at the constant reference temperature $T_{0}$ and is subject to a ramp type increase in load. Mathematically, these boundary conditions can be written as

$$
\begin{gathered}
\phi(0, t)=0, \\
\sigma(0, t)=-\sigma^{*} h(t)-p,
\end{gathered}
$$

where $\sigma^{*}$ is a constant load and $h(t)$ is a function as defined by (45). Laplace transform of the equations (54) containing boundary conditions yields (in non-dimensional form)

$$
\begin{gathered}
\bar{\phi}(0, s)=\bar{\phi}_{0}=0 \\
\bar{\sigma}(0, s)=\bar{\sigma}_{0}=-\sigma^{*} \alpha_{3}-\frac{\delta_{1}}{s} .
\end{gathered}
$$

Adopting the same procedure, the expressions for all the considered physical quantities can be found as

$$
\begin{gathered}
\bar{\phi}=\frac{1}{\left(\lambda_{1}-\lambda_{2}\right)}\left[\left\{L_{2}\left(\sigma^{*} \alpha_{3}+\frac{\delta_{1}}{s}-\alpha_{2}\right)+\alpha_{1}\left(\lambda_{1}-L_{1}\right)\right\} e^{-\sqrt{\lambda_{2}} x}\right. \\
-\left\{L_{2}\left(\sigma^{*} \alpha_{3}+\frac{\delta_{1}}{s}-\alpha_{2}\right)\right. \\
\left.\left.+\alpha_{1}\left(\lambda_{2}-L_{1}\right)\right\} e^{-\sqrt{\lambda_{1}} x}\right]-\alpha_{1}
\end{gathered}
$$

$$
\begin{gathered}
\bar{\sigma}=\frac{1}{\left(\lambda_{1}-\lambda_{2}\right)}\left[\left\{\left(\lambda_{1}-M_{2}\right)\left(\alpha_{2}-\sigma^{*} \alpha_{3}-\frac{\delta_{1}}{s}\right)\right.\right. \\
\left.-\alpha_{1} M_{1}\right\} e^{-\sqrt{\lambda_{2}} x} \\
-\left\{\left(\lambda_{2}-M_{2}\right)\left(\alpha_{2}-\sigma^{*} \alpha_{3}-\frac{\delta_{1}}{s}\right)\right. \\
\left.\left.-\alpha_{1} M_{1}\right\} e^{-\sqrt{\lambda_{1}} x}\right]-\alpha_{2},
\end{gathered}
$$




$$
\begin{aligned}
& \bar{\theta}=\frac{1}{\left(\lambda_{1}-\lambda_{2}\right)}\left[\left\{L_{2}\left(\sigma^{*} \alpha_{3}+\frac{\delta_{1}}{s}-\alpha_{2}\right)+\alpha_{1}\left(\lambda_{1}-L_{1}\right)\right\}\right. \\
& \times\left(1-\beta \lambda_{2}\right) e^{-\sqrt{\lambda_{2}} x} \\
& -\left\{L_{2}\left(\sigma^{*} \alpha_{3}+\frac{\delta_{1}}{s}-\alpha_{2}\right)+\alpha_{1}\left(\lambda_{2}-L_{1}\right)\right\} \\
& \left.\times\left(1-\beta \lambda_{1}\right) e^{-\sqrt{\lambda_{1}} x}\right]-\alpha_{1} \\
& \bar{e}=\frac{1}{\left(\lambda_{1}-\lambda_{2}\right)}\left[\left\{\left(\sigma^{*} \alpha_{3}+\frac{\delta_{1}}{s}-\alpha_{2}\right)\right.\right. \\
& \times\left\{\varepsilon_{1} L_{2}\left(1-\beta \lambda_{2}\right)-\left(\lambda_{1}-M_{2}\right)\right\} \\
& \left.+\alpha_{1}\left\{\varepsilon_{1}\left(\lambda_{1}-L_{1}\right)\left(1-\beta \lambda_{2}\right)-M_{1}\right\}\right\} e^{-\sqrt{\lambda_{2}} x} \\
& -\left\{\left(\sigma^{*} \alpha_{3}+\frac{\delta_{1}}{s}-\alpha_{2}\right)\right. \\
& \times\left\{\varepsilon_{1} L_{2}\left(1-\beta \lambda_{1}\right)-\left(\lambda_{2}-M_{2}\right)\right\} \\
& \left.+\alpha_{1}\left\{\varepsilon_{1}\left(\lambda_{2}-L_{1}\right)\left(1-\beta \lambda_{1}\right)-M_{1}\right\}\right\} \\
& \left.x e^{-\sqrt{\lambda_{1}} x}\right]-\varepsilon_{1} \alpha_{1}-\alpha_{2}+\frac{\delta_{1}}{s} \\
& \bar{u}=-\frac{1}{\alpha s^{2}\left(\lambda_{1}-\lambda_{2}\right)}\left[\sqrt { \lambda _ { 2 } } \left\{\left(\lambda_{1}-M_{2}\right)\left(\alpha_{2}-\sigma^{*} \alpha_{3}-\frac{\delta_{1}}{s}\right)\right.\right. \\
& \left.-\alpha_{1} M_{1}\right\} e^{-\sqrt{\lambda_{2}} x} \\
& -\sqrt{\lambda_{1}}\left\{\left(\lambda_{2}-M_{2}\right)\left(\alpha_{2}-\sigma^{*} \alpha_{3}-\frac{\delta_{1}}{s}\right)\right. \\
& \left.\left.-\alpha_{1} M_{1}\right\} e^{-\sqrt{\lambda_{1}} x}\right] \text {. }
\end{aligned}
$$

\section{Limiting Cases}

4.1. Case I: With One Temperature. The expressions for different distributions in the above two sets of boundary conditions with one temperature, that is, the case when conductive temperature coincides with thermodynamical temperature, can be obtained from expressions (47)-(50), (53), and (56) by setting $a=0$ and consequently $\beta=0$ in the field equations.

4.2. Case II: Without Magnetic Effect. In order to discuss the problem of wave propagation in two-temperature thermoelasticity with initial stress, it is sufficient to set $H_{0}=0$, and accordingly, we have $\alpha=1$. Taking into consideration the above-mentioned modifications, the corresponding expressions of the physical fields can be obtained from expressions (47)-(50), (53), and (56).
4.3. Case III: Without Initial Stress. The required expressions for the components of displacement, stress, strain, and temperature can be procured from the expressions (47)-(50), (53), and (56) in the absence of initial stress by putting $\eta=1$ and $p=0$ in the governing equation. In addition to this if we also neglect magnetic effect (i.e., $H_{0}=0$ ), then our results match with those obtained by Youssef and Al-Lehaibi [28].

\section{Numerical Inversion of the Laplace Transform}

In the previous section, we obtained the solutions of two problems in the Laplace transform domain $(x, s)$. We shall now outline briefly the numerical inversion method used to find the solution in the physical domain. Let $\bar{f}(s)$ be the Laplace transform of a function $f(t)$. The inversion formula for Laplace transform can be written as Churchill [33]

$$
f(t)=\frac{1}{2 \pi \iota} \int_{v-\iota \infty}^{v+\iota \infty} e^{s t} \bar{f}(s) d s
$$

where $v$ is an arbitrary real number greater than all the real parts of the singularities of $\bar{f}(s)$. Taking $s=v+\imath w$ and using Fourier series in the interval $[0,2 T]$, we obtain the approximate formula Honig and Hirdes [31]

$$
f(t) \cong f_{N}(t)=\frac{1}{2} c_{0}+\sum_{k=1}^{N} c_{k}, \quad \text { for } 0 \leq t \leq 2 T,
$$

where

$$
c_{k}=\frac{e^{v t}}{T} \operatorname{Re}\left[e^{\imath k \pi t / T} \bar{f}\left(v+\frac{\imath k \pi}{T}\right)\right] .
$$

Two methods are used to reduce the total error. First, the "Korrecktur" method is used to reduce the discretization error. Next, the $\varepsilon$-algorithm is used to reduce the truncation error and hence to accelerate convergence. The details of these methods can be found in Honig and Hirdes [31]. The values of $v$ and $T$ are chosen according the criteria outlined in Honig and Hirdes [31].

\section{Numerical Results and Discussions}

With an aim to illustrate the contribution of initial stress, two temperatures, and magnetic field on the field quantities, a numerical analysis is carried out. The material chosen for the purpose of numerical computation is copper for which

$$
\begin{gathered}
E=36.9 \times 10^{10} \mathrm{~kg} \mathrm{~m}^{-1} \mathrm{~s}^{-2}, \quad v=0.33, \quad T_{0}=293 \mathrm{~K}, \\
\rho=8954 \mathrm{~kg} \mathrm{~m}^{-3}, \quad k=386 \mathrm{Wm}^{-1} \mathrm{~K}^{-1}, \\
\mathcal{c}_{E}=383.1 \mathrm{~J} \mathrm{~kg}^{-1} \mathrm{~K}^{-1}, \\
\tau_{0}=0.02 \mathrm{~s}, \quad \alpha_{t}=1.78 \times 10^{-5} \mathrm{~K}^{-1}, \\
H_{0}=\left(10^{7} / 4 \pi\right) \mathrm{Am}^{-1}, \\
\varepsilon_{0}=\left(10^{-9} / 36 \pi\right) \mathrm{Fm}^{-1}, \quad \mu_{0}=4 \pi(10)^{-7} \mathrm{Hm}^{-1} .
\end{gathered}
$$


The relation $E / \mu=2(1+v)$ connecting Young's modulus $(E)$, the modulus of rigidity $(\mu)$, and Poisson's ratio $(v)$ for isotropic elastic solids does not hold well for real earthy materials, namely, sand, and soil. Weiskopf [34] has established a stress-strain relation for the same. He has investigated that due to the slipping of granules on each other, the resistance to shear is much less than in a solid and the resulting shearing deflection is much greater. For these materials $E / \mu>2(1+v)$. So the relation $E / \bar{\mu}=2 \eta(1+v)$ has been considered here. Also the other generalized Lame's constant may be defined as

$$
\bar{\lambda}=\frac{E v}{\eta(1+v)(1-2 v)},
$$

where $\eta(\geq 1)$ is defined as sandiness or initial stress parameter. $\eta=1$ corresponds to isotropic elastic medium with no initial stress.

The values of other parameters arising in the present analysis are taken as

$$
t_{0}=0.5, \quad \sigma^{*}=1, \quad \phi^{*}=1, \quad \beta=0.1 .
$$

With these numerical values of the parameters, the values of the field quantities are computed for the time $t=0.1$. All the field quantities have been examined for the following four cases: (i) initially stressed magneto-thermoelastic solid with two temperatures (ISMTT, $\eta=2.5, p=1$, and $\beta=0.1$ ), (ii) initially stressed magneto-thermoelastic solid (ISMT, $\eta=2.5$, $p=1$, and $\beta=0$ ), (iii) magneto-thermoelastic solid with two temperatures (MTT, $\eta=1, p=0$, and $\beta=0.1$ ), and (iv) initially stressed thermoelastic solid with two temperatures (ISTT, $\eta=2.5, p=1, \beta=0.1$, and $H_{0}=0$ ).

From application point of view, we have divided the graphs into three groups. The first group (Figures 1-4) represents the distribution of variables for the case of ramp type increase in boundary load. The variation of the field variables for the case of ramp type increase in boundary temperature is observed in second group (Figures 5-8). In this group, we have not considered the ISTT case (i.e., without magnetic effect) graphically keeping in view the resolution and clarity of plots. However, magnetic field has the same effect on the physical fields as in the case of ramp type increase in boundary load. The third group (Figures 9-12) displays the influence of ramp parameter on the considered physical variables for three values of $t_{0}$ which are taken as $0.1,0.5$, and 0.9 .

6.1. Boundary Load. Figure 1 displays a comparison of displacement component in the context of four cases. The displacement field shows maximum negative value at the boundary of the half-space and then changes to zero value with increase in the spatial coordinate. Also the effects of two temperature, magnetic field, and initial stress parameter are very much pronounced. The two-temperature parameter has decreased the magnitude of the displacement field, while initial stress parameter and magnetic field have increased the magnitude. Figure 2 exhibits the spatial variation of the thermodynamical temperature $\theta$. The thermodynamical temperature is enjoying the similar pattern in all the four

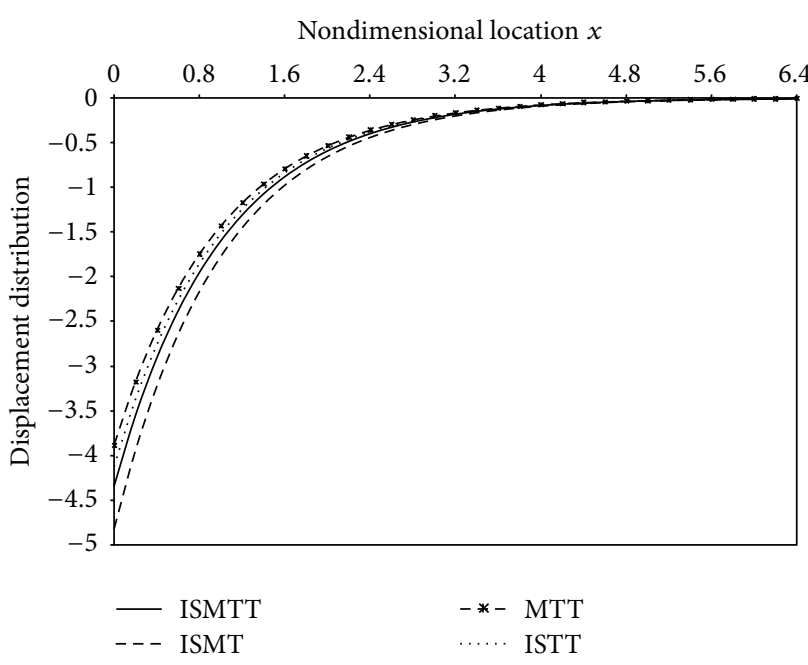

FIgURE 1: Profile of displacement distribution (boundary load).

cases except that the magnitudes in all the cases are different. In case of ISMT, it starts with zero value and further decreases continuously to a maximum negative value -1.8108 at $x=1$. Afterwards, its value increases and attains the zero value. Significant difference is noticed in case of MTT. Also the two-temperature parameter is playing a vital role. It has both increasing and decreasing effects on the thermodynamical temperature. It can also be seen from the plot that magnetic field acts to increase the magnitude of thermodynamical temperature.

The variation of stress component $\sigma$ with spatial coordinate $x$ has been observed in Figure 3. The stress component starts with positive values in all the cases, and it decreases continuously to attain its lowest value. Presence of initial stress, two temperature parameter, and magnetic field has appreciably increased the magnitude of the stress component. Another interesting observation is the nonvanishing behaviour of the stress component in ISMTT, ISMT, and ISTT cases, though the values are very small. This important phenomenon has happened owing to the presence of initial stress. Figure 4 is plotted to describe conductive temperature $\phi$ for all the four cases. The conductive temperature starts with a zero value for all cases and has decreasing behaviour for the interval $0 \leq x \leq 1$. It attains its highest negative values $-1.7235,-1.8108,-4.2750$, and -1.6373 at $x=1$ for the ISMTT, ISMT, MTT, and ISTT cases, respectively. Initial stress parameter has strongly influenced the conductive temperature. It is also apparent from the figure that two temperature parameter and magnetic field have also produced significant difference in conductive temperature.

6.2. Boundary Temperature. Figure 5 is drawn to show the variation of the displacement component with distance $x$. The displacement component starts with a positive maximum value and then decreases monotonically with $x$ attaining its lowest value at the location $x=2$ and then gradually diminishes to zero. Here the location at which the extreme values are attained is independent of the initial stress and two 
Nondimensional location $x$

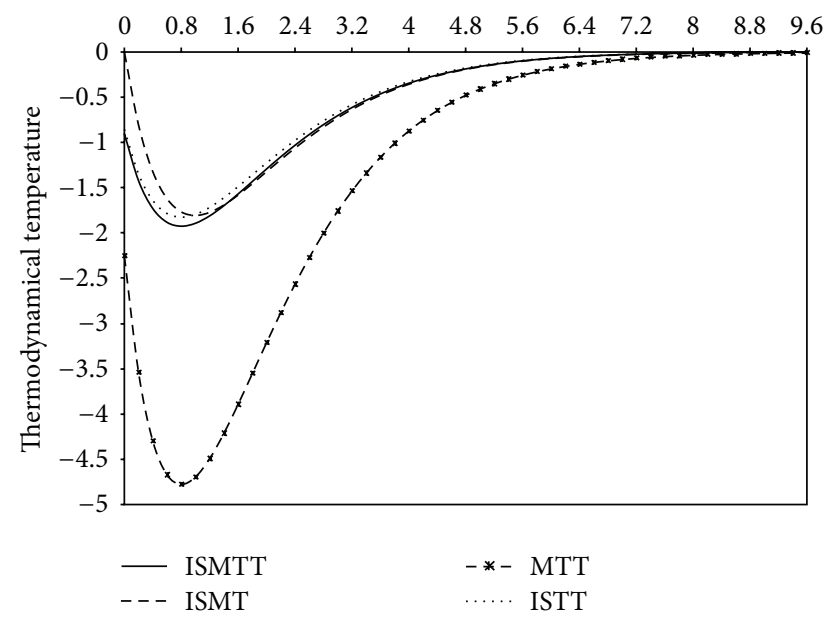

FIGURE 2: Profile of thermodynamical temperature (boundary load).

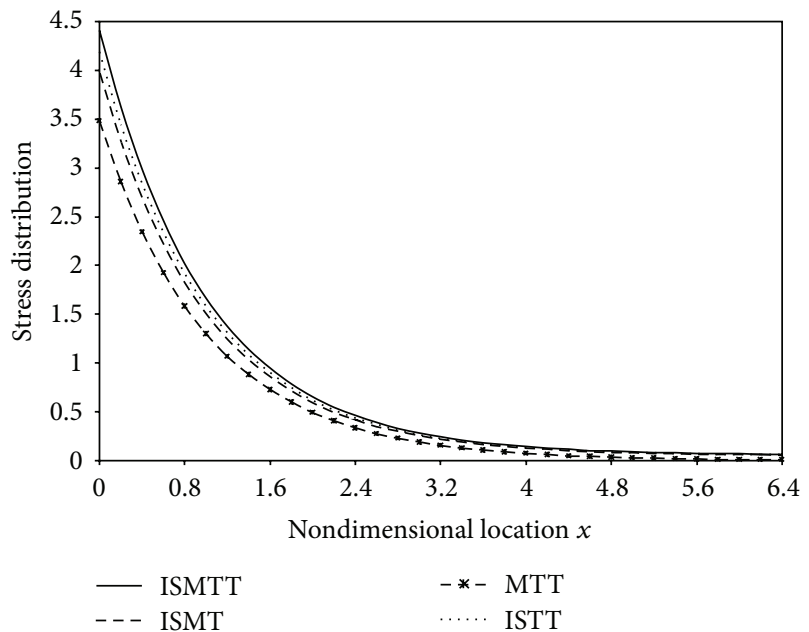

FIGURE 3: Profile of stress distribution (boundary load).

Nondimensional location $x$

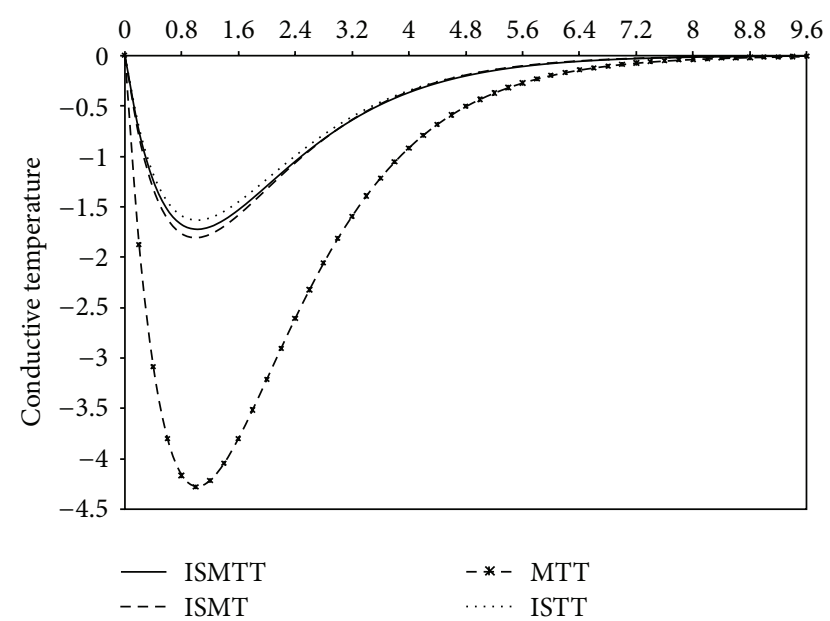

Figure 4: Profile of conductive temperature (boundary load).

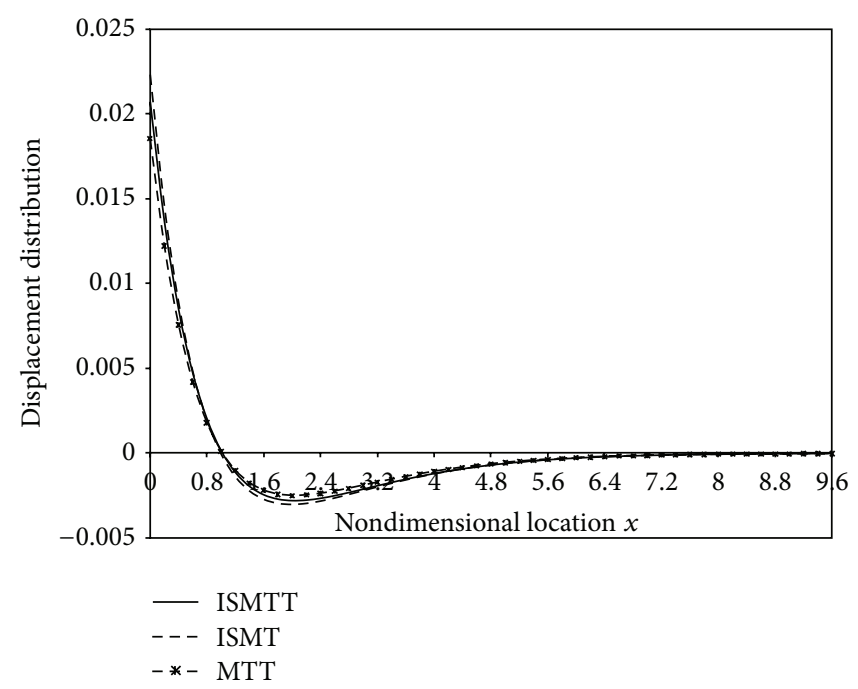

FIGURE 5: Profile of displacement distribution (boundary temperature).

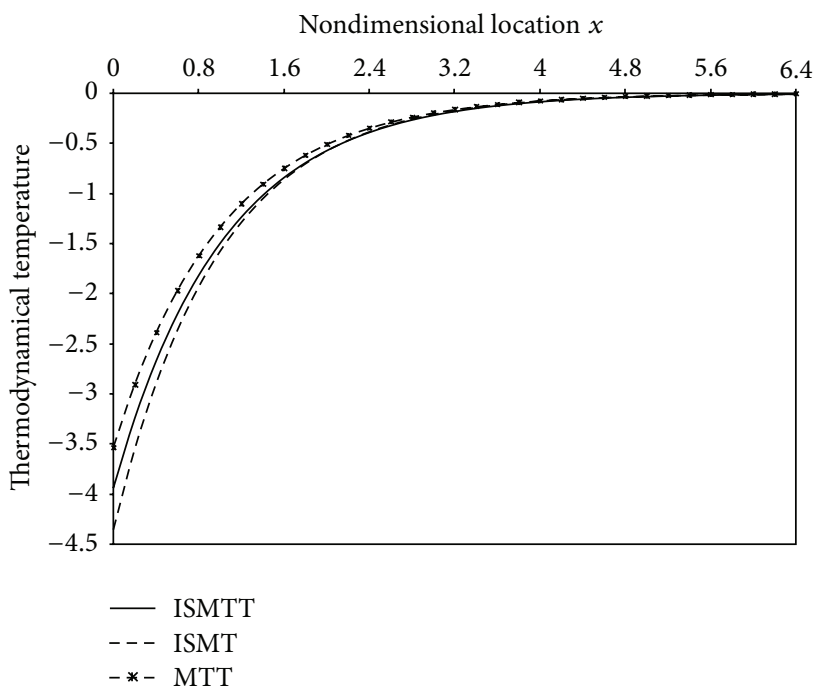

FIgure 6: Profile of thermodynamical temperature (boundary temperature).

temperature parameter. In fact the effect of the stress and two temperature parameter is very tiny, and this effect vanishes with the non-dimensional location. Figure 6 represents the change in the thermodynamical temperature $\theta$ with location $x$. The values of the thermodynamical temperature are found to be small in magnitude in MTT case. It can also be seen from the figure that the trend of thermodynamical temperature is similar in all the three cases.

Figure 7 gives the stress variation in the direction of wave propagation. At $x=0$, the stress reduces to zero for the MTT case which agrees with the boundary condition. In this case also, the stress is having nonvanishing behaviour with very small magnitudes for the ISMTT and ISMT cases. The influence of initial stress parameter is very much pronounced on the stress component. In Figure 8, we have depicted 


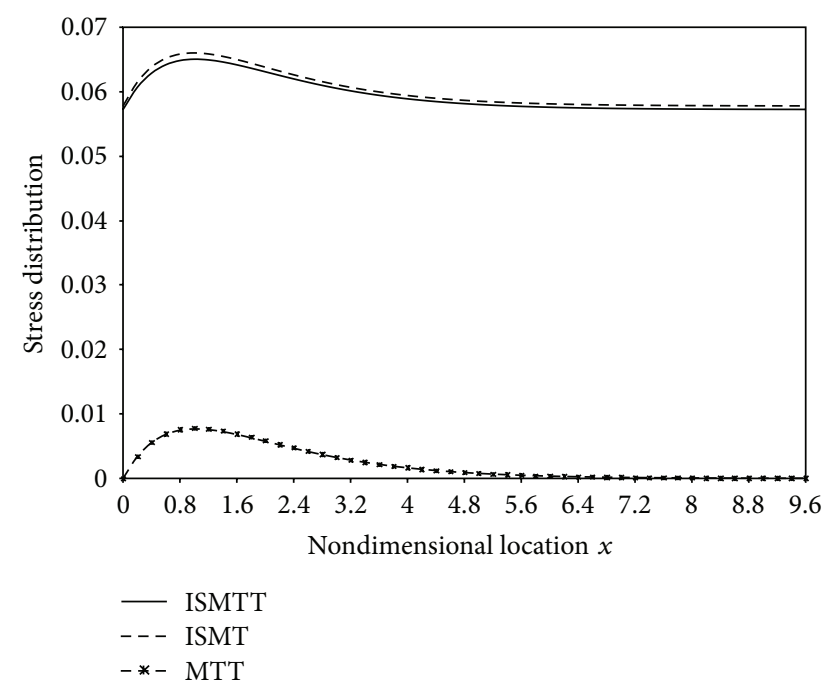

Figure 7: Profile of stress distribution (boundary temperature).

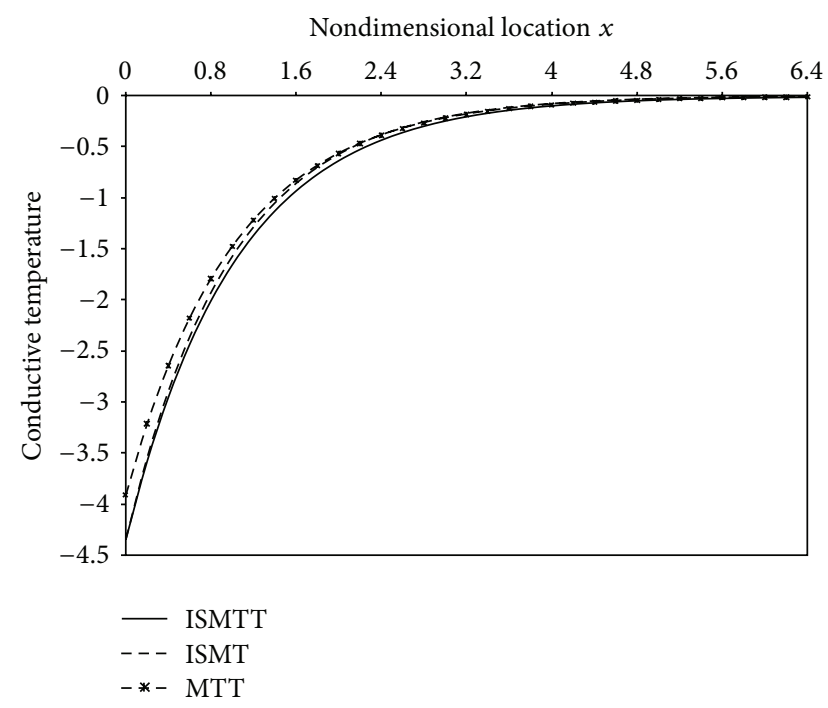

Figure 8: Profile of conductive temperature (boundary temperature).

the profile of conductive temperature $\phi$ with location $x$. It is behaving like an increasing function in the entire range for all the three cases. Also the contribution of additional parameters like initial stress, two-temperature, and magnetic field reduces to zero with increase in the spatial coordinate.

6.3. Influence of Ramp Parameter (Boundary Load). Figures 9-12 display the values of the considered physical variables in the direction of wave propagation for three values of ramp parameter $t_{0}$ which are taken to be $0.1,0.5$, and 0.9 . We observe from these figures that all the variables behave in the same manner due to the change in the values of ramp parameter with some difference in magnitudes. Increase in the value of ramp parameter is making the magnitudes of the field variables small. Hence the ramp parameter has a salient effect on all the variables.

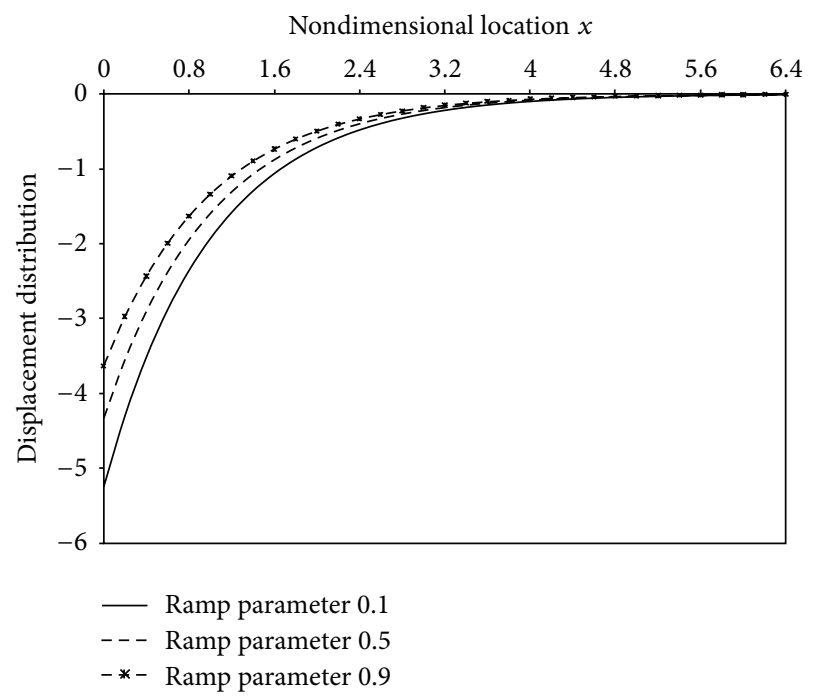

FIGURE 9: Influence of ramp parameter on displacement distribution.

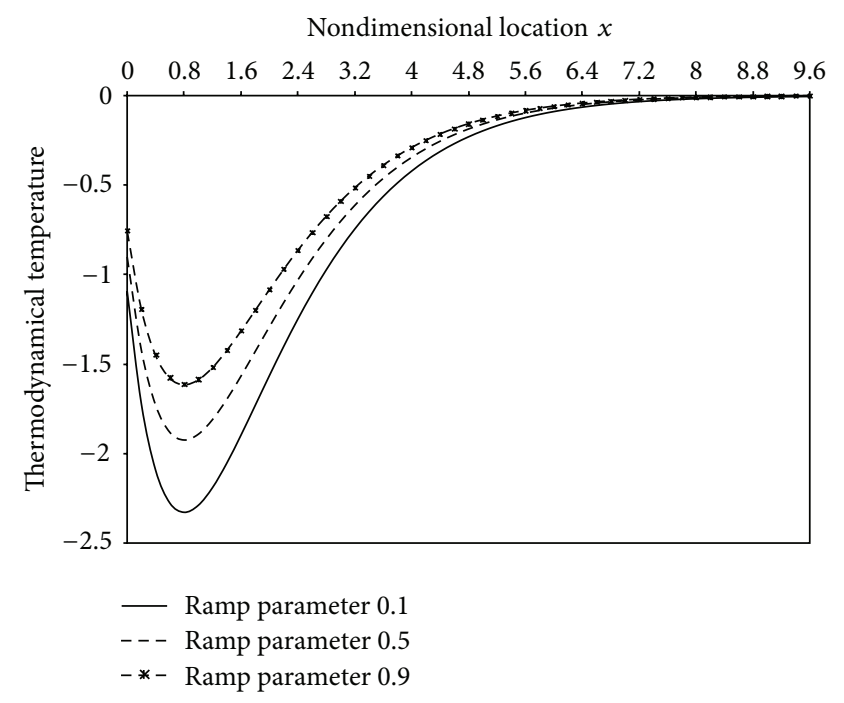

FIGURE 10: Influence of ramp parameter on thermodynamical temperature.

\section{Conclusions}

A mathematical study is made to discuss the problem of magneto-thermoelastic interactions in two-temperature theory with hydrostatic initial stress. The theoretical and numerical results reveal that all the four parameters, namely, initial stress, two temperature, magnetic field, and ramp parameter have significant effect on the considered physical variables. It is concluded the following.

(1) The phenomenon of finite speed of propagation is manifested in all the figures except the stress component which is also having very small value.

(2) With the increase in distance, the results are quite close to each other, which is in agreement with the generalized theories of thermoelasticity. 


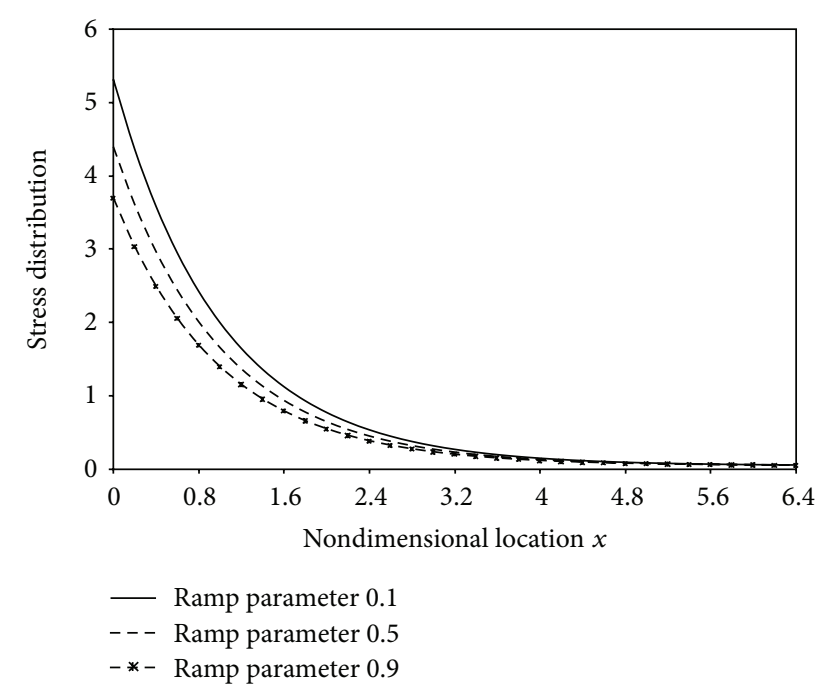

FIGURE 11: Influence of ramp parameter on stress distribution.

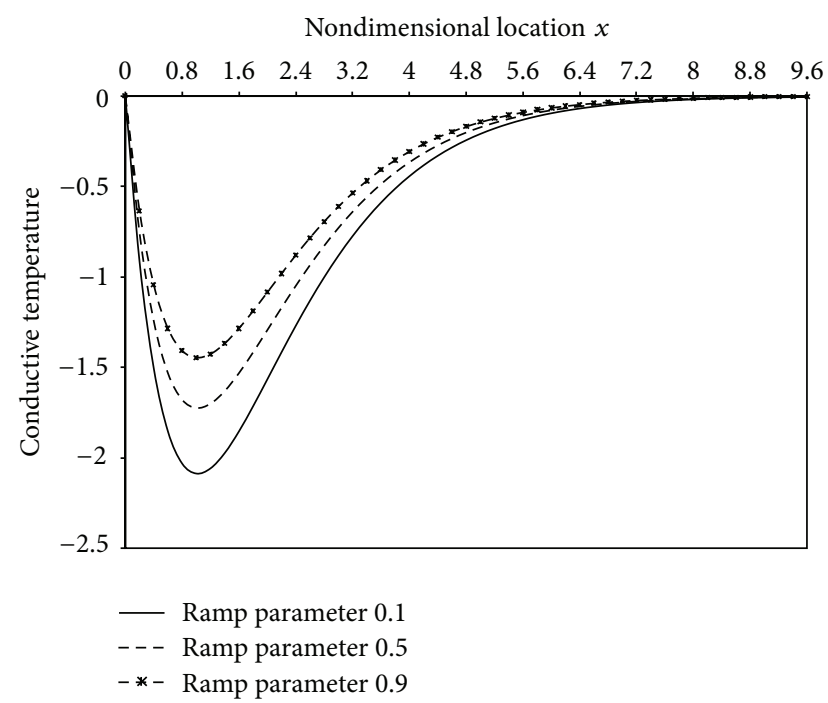

FIGURE 12: Influence of ramp parameter on conductive temperature.

(3) It is apparent from the figures that initial stress parameter strongly affects the physical quantities.

(4) The nature of the source (boundary load or temperature) highly influences the physical quantities. The body is deformed to a large extent when a boundary load is applied. The displacement component exhibits increasing behaviour in case of boundary load, while in case of boundary temperature, it initially decreases to its maximum negative value and then gradually approaches to zero. Similarly, the other physical quantities behave significantly different when different type of sources are applied.

(5) All the physical variables are observed to be sensitive (more or less) towards the two temperature parameter.
(6) Presence of magnetic field has affected all the considered physical variables. It acts to increase the magnitude of all the field variables.

(7) The ramp parameter has significantly affected all the studied fields. Increment in the value of ramp parameter causes decrement in the values of all the fields which is very obvious from the figures.

(8) All the functions except stress vanish identically outside some finite interval. This is not the case in coupled thermoelasticity where the considered functions have non-vanishing values for all $x$ due to the infinite speed of propagation of heat waves.

This research demonstrates how state space approach can be extended to study the problem involving magnetothermoelastic interactions in two temperature theory with initial stress which has been rarely employed in analyzing such problems. The inclusion of magnetic and initial stress parameters provides a more realistic model of the generalized thermoelasticity theory.

\section{Nomenclature}

$\bar{\lambda}, \bar{\mu}$ : Counterparts of Lame's parameters

$t_{0}$ : Ramp parameter

$p$ : Initial pressure

$\eta$ : Initial stress parameter

$E$ : Young's modulus

$v$ : Poisson's ratio

a: $\quad$ Two temperature parameter

$\alpha_{t}$ : Coefficient of linear thermal expansion

$T$ : Thermodynamical temperature

$T_{0}$ : Temperature of the medium in its natural state assumed to be $\left|\left(T-T_{0}\right) / T_{0}\right| \ll 1$

$\phi$ : Conductive temperature

$\sigma_{i j}:$ Components of the stress tensor

$u_{i}$ : Components of the displacement vector

$\rho$ : Density of the medium

$e_{i j}$ : Components of the strain tensor

$e: \quad$ Cubical dilatation

$c_{E}$ : Specific heat at constant strain

$k: \quad$ Thermal conductivity

$\tau_{0}$ : Thermal relaxation time

$\mu_{0}$ : Magnetic permeability

$\varepsilon_{0}: \quad$ Electric permittivity

$F_{i}$ : Lorentz force

$\beta_{1}: \quad(3 \bar{\lambda}+2 \bar{\mu}) \alpha_{t}$

$\delta_{i j}:$ Kronecker delta function.

\section{References}

[1] M. A. Biot, "Thermoelasticity and irreversible thermodynamics," Journal of Applied Physics, vol. 27, no. 3, pp. 240-253, 1956.

[2] H. W. Lord and Y. Shulman, "A generalized dynamical theory of thermoelasticity," Journal of the Mechanics and Physics of Solids, vol. 15, no. 5, pp. 299-309, 1967.

[3] A. E. Green and K. A. Lindsay, “Thermoelasticity," Journal of Elasticity, vol. 2, no. 1, pp. 1-7, 1972. 
[4] D. S. Chandrasekharaiah, "Thermoelasticity with second sound: a review," Applied Mechanics Reviews, vol. 39, pp. 355-376, 1986.

[5] D. S. Chandrasekharaiah, "Hyperbolic thermoelasticity: a review of recent literature," Applied Mechanics Reviews, vol. 51, pp. 705-729, 1998.

[6] G. Paria, "On magneto-thermoelastic plane waves," Proceedings of the Cambridge Philosophical Society, vol. 58, pp. 527-531, 1962.

[7] A. H. Nayfeh and S. Nemat-Nasser, "Electromagneto-thermoelastic plane waves in solids with thermal relaxation," Journal of Applied Mechanics, vol. 39, no. 1, pp. 108-113, 1972.

[8] S. K. R. Choudhuri, "Electro-magneto-thermo-elastic plane waves in rotating media with thermal relaxation," International Journal of Engineering Science, vol. 22, no. 5, pp. 519-530, 1984.

[9] M. A. Ezzat, "Generation of generalized magnetothermoelastic waves by thermal shock in a perfectly conducting half-space," Journal of Thermal Stresses, vol. 20, no. 6, pp. 617-633, 1997.

[10] M. A. Ezzat, M. I. Othman, and A. A. Smaan, "State space approach to two-dimensional electromagneto-thermoelastic problem with two relaxation times," International Journal of Engineering Science, vol. 39, no. 12, pp. 1383-1404, 2001.

[11] L. Y. Bahar and R. B. Hetnarski, "State space approach to thermoelasticity," Journal of Thermal Stresses, vol. 2, no. 1, pp. 135-145, 1978.

[12] H. H. Sherief, "State space formulation for generalized thermoelasticity with one relaxation time including heat sources," Journal of Thermal Stresses, vol. 16, pp. 163-180, 1993.

[13] T. He, X. Tian, and Y. P. Shen, "State space approach to one-dimensional thermal shock problem for a semi-infinite piezoelectric rod," International Journal of Engineering Science, vol. 40, no. 10, pp. 1081-1097, 2002.

[14] M. A. Ezzat, "State space approach to solids and fluids," Canadian Journal of Physics, vol. 86, no. 11, pp. 1241-1250, 2008.

[15] H. M. Youssef and A. A. El-Bary, "Generalized thermoelastic infinite layer subjected to ramp-type thermal and mechanical loading under three theories-State space approach," Journal of Thermal Stresses, vol. 32, no. 12, pp. 1293-1309, 2009.

[16] K. A. Elsibai and H. M. Youssef, "State-space approach to vibration of gold nano-beam induced by ramp type heating without energy dissipation in femtoseconds scale," Journal of Thermal Stresses, vol. 34, no. 3, pp. 244-263, 2011.

[17] S. Deswal, S. S. Sheoran, and K. K. Kalkal, "The effect of magnetic field and initial stress on fractional order generalized thermoelastic half-space," Journal of Mathematics, vol. 2013, Article ID 489863, 11 pages, 2013.

[18] M. A. Biot, Mechanics of Incremental Deformations, John Wiley \& Sons, New York, NY, USA, 1965.

[19] A. Chattopadhyay, S. Bose, and M. Chakraborty, "Reflection of elastic waves under initial stress at a free surface: P and SV motion," Journal of the Acoustical Society of America, vol. 72, no. 1, pp. 255-263, 1982.

[20] A. Montanaro, "On singular surfaces in isotropic linear thermoelasticity with initial stress," Journal of the Acoustical Society of America, vol. 106, no. 3 I, pp. 1586-1588, 1999.

[21] M. I. A. Othman and Y. Song, "Reflection of plane waves from an elastic solid half-space under hydrostatic initial stress without energy dissipation," International Journal of Solids and Structures, vol. 44, no. 17, pp. 5651-5664, 2007.

[22] B. Singh, "Effect of hydrostatic initial stresses on waves in a thermoelastic solid half-space," Applied Mathematics and Computation, vol. 198, no. 2, pp. 494-505, 2008.
[23] P. J. Chen and M. E. Gurtin, "On a theory of heat conduction involving two temperatures," Zeitschrift für angewandte Mathematik und Physik, vol. 19, no. 4, pp. 614-627, 1968.

[24] P. J. Chen, M. E. Gurtin, and W. O. Williams, "A note on nonsimple heat conduction," Zeitschrift für angewandte Mathematik und Physik, vol. 19, pp. 969-970, 1968.

[25] P. J. Chen, M. E. Gurtin, and W. O. Williams, "On the thermodynamics of non-simple elastic materials with two temperatures," Zeitschrift für angewandte Mathematik und Physik, vol. 20, no. 1, pp. 107-112, 1969.

[26] R. Quintanilla, "On existence, structural stability, convergence and spatial behavior in thermoelasticity with two temperatures," Acta Mechanica, vol. 168, no. 1-2, pp. 61-73, 2004.

[27] H. M. Youssef, "Theory of two-temperature-generalized thermoelasticity," IMA Journal of Applied Mathematics, vol. 71, no. 3, pp. 383-390, 2006.

[28] H. M. Youssef and E. A. Al-Lehaibi, "State-space approach of two-temperature generalized thermoelasticity of onedimensional problem," International Journal of Solids and Structures, vol. 44, no. 5, pp. 1550-1562, 2007.

[29] H. M. Youssef and A. A. El-Bary, “Two-temperature generalized thermoelasticity with variable thermal conductivity," Journal of Thermal Stresses, vol. 33, no. 3, pp. 187-201, 2010.

[30] J. C. Misra, N. C. Chattopadhyay, and A. Chakravorty, "Study of thermoelastic wave propagation in a half-space using GN theory," Journal of Thermal Stresses, vol. 23, no. 4, pp. 327-351, 2000.

[31] G. Honig and U. Hirdes, "A method for the numerical inversion of Laplace transforms," Journal of Computational and Applied Mathematics, vol. 10, no. 1, pp. 113-132, 1984.

[32] G. C. Charles, Matrices and Linear Transformations, AddisonWesley, Reading, Mass, USA.

[33] R. Churchill, Operational Mathematics, McGraw-Hill, New York, NY, USA, 1972.

[34] W. H. Weiskopf, "Stresses in soils under a foundation," Journal of the Franklin Institute, vol. 239, no. 6, pp. 445-465, 1945. 

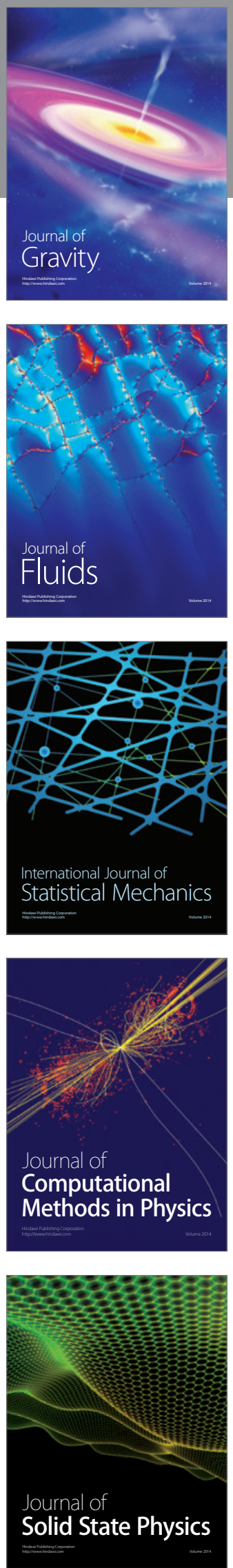

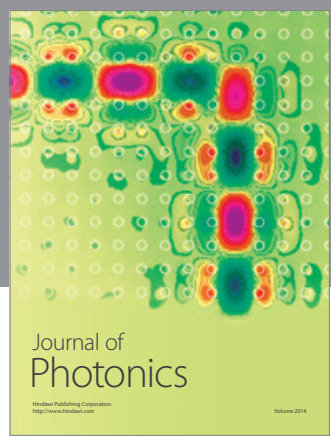

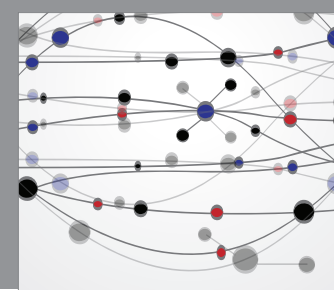

The Scientific World Journal

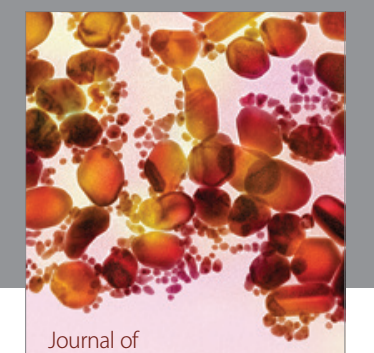

Soft Matter
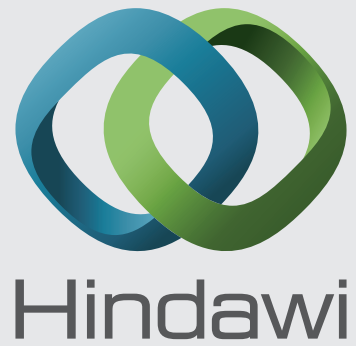

Submit your manuscripts at

http://www.hindawi.com
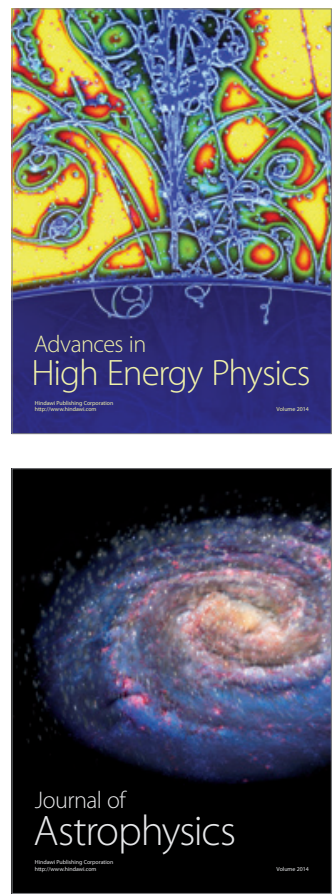
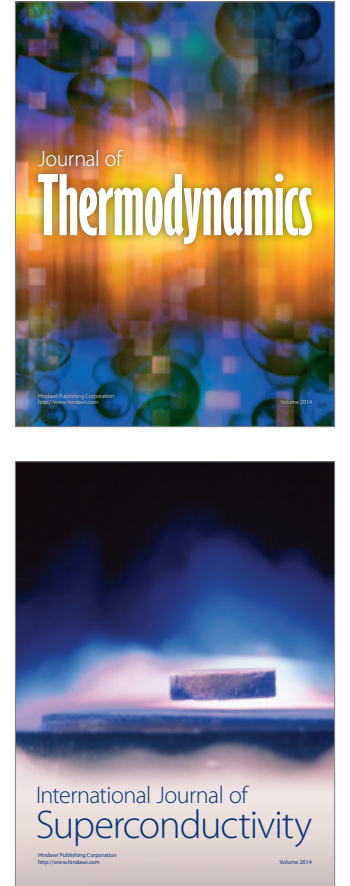
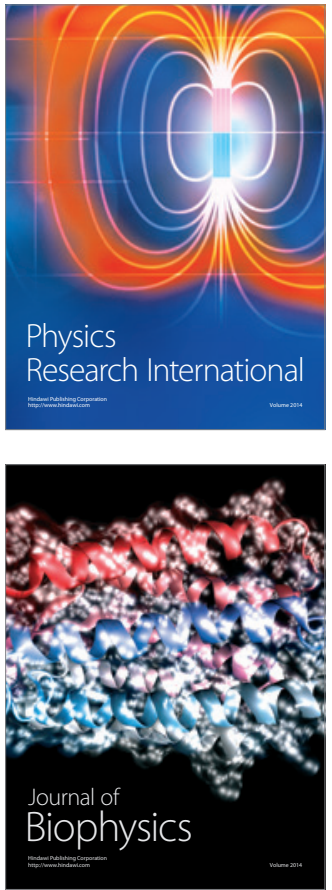
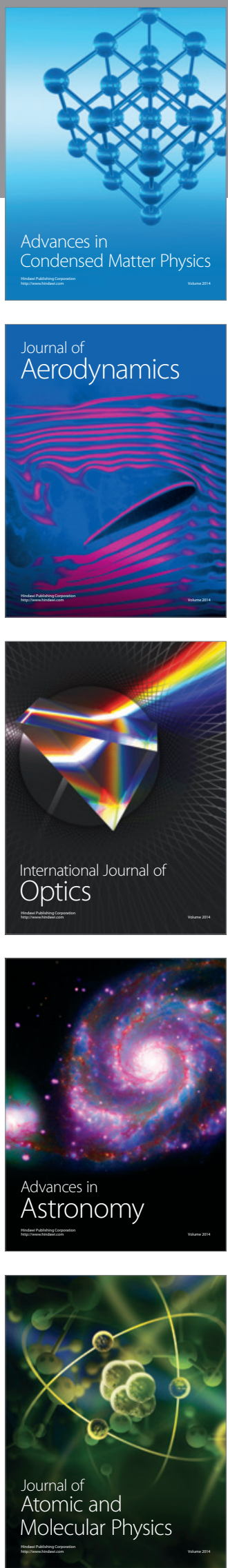